\title{
Evaluation of Tadalafil Nanosuspensions and Their PEG Solid Dispersion Matrices for Enhancing Its Dissolution Properties
}

\author{
Wasfy M. Obeidat ${ }^{1,3}$ and Al-Sayed A. Sallam ${ }^{2}$
}

\begin{abstract}
Received 28 August 2013; accepted 14 December 2013; published online 9 January 2014

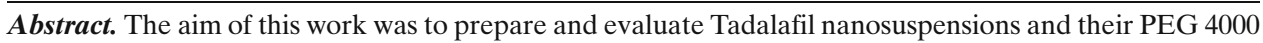
solid dispersion matrices to enhance its dissolution rate. Nanosuspensions were prepared by precipitation/ ultrasonication technique at $5^{\circ} \mathrm{C}$ where different stabilizers were screened for stabilization. Nanosuspensions were characterized in terms of particle size and charge. Screening process limited suitable stabilizers into structurally related surfactants composed of a mixture of Tween 80 and Span80 at 1:1 ratio (in percent, weight/volume) in adjusted alkaline $\mathrm{pH}$ (named TDTSp-OH). The surfactant mixture aided the production of nanosuspensions with an average particle size of $193 \pm 8 \mathrm{~nm}$ and with short-term stability sufficient for further processing. Solid dispersion matrices made of dried Tadalafil nanosuspensions or dried Tadalafil raw powder suspensions and PEG 4000 as a carrier were prepared by direct compression. Drying was performed via dry heat or via freeze dry. Drug release studies showed that, in general, tablet formulations made of freeze-dried product exhibited faster initial release rates than the corresponding tablets made of oven-dried products which could be attributed to possible larger crystal growth and larger crushing strengths of oven-dried formulations. At best, $60 \%$ of drug was released from solid dispersion matrices, while more than $90 \%$ of drug was released from TDTSp-OH nanosuspension within the first 5 min. In conclusion, Tadalafil nanosuspensions obtained using a mixed surfactant system provided rapid dissolution rates of Tadalafil that can theoretically enhance its bioavailability.
\end{abstract}

KEY WORDS: nanosuspension; particle size; solid dispersion; stabilizer; tablets; Tadalafil.

\section{INTRODUCTION}

A sharp increase in introduction of new highly lipophilic (poor aqueous solubility) is experienced nowadays $(1,2)$ leading to poor oral bioavailability due to low dissolution rates in the gastrointestinal tract (3). This ends up with lowering of the efficacy of these poorly soluble drugs since dissolution is considered the first step in the absorption process (4).

Solubilization via certain techniques such as salt formation, surfactant, cosolvents, polymers, lipids, complexing agent and prodrugs was employed to improve the solubility and hence the bioavailability of poorly soluble drugs $(5,6)$. However, these methods, not unexpectedly, suffer several drawbacks $(7,8)$.

Micronization is another technique employed for improving drug dissolution rates, but was found to be insufficient to overcome bioavailability problems of many poorly soluble drugs. Therefore, a consequent step was to shift to nanosization since saturation solubility and dissolution rate can be enhanced according to Noyes-Whitney equation (9). In 1990s, Elan Nanosystems (San Francisco, CA, USA)

\footnotetext{
${ }^{1}$ Department of Pharmaceutical Technology, Jordan University of Science and Technology, Irbid 22110, P.O. Box 3030, Jordan.

${ }^{2}$ TQ Pharma, Amman 11947, P. O. Box 1019, Jordan.

${ }^{3}$ To whom correspondence should be addressed. (e-mail: wasfy72@yahoo.com)
}

revolutionized the use of nanocrystals instead of microcrystals for oral bioavailability enhancement and nanosuspensions for intravenous or pulmonary drug delivery. However, it should be stated that although poor drug solubility in aqueous medium lowers its oral bioavailability, nanosizing does not guarantee high bioavailability $(3,10)$. Nevertheless, in recent years, nanoparticle or nanocrystal engineering processes have become promising approaches for the enhancement of dissolution rates of poorly aqueous soluble drugs (11-15).

The preparation of drug nanoparticles can be done through top-down or bottom-up approaches. The anti-solvent precipitation method produced by List and Sucker in 1990s (16) is a bottom-up process and was adopted in the current work in the presence of surfactants, polymers or a mixture of both as stabilizers. The method involves cavitation via ultrasonication. It is relatively rapid and of low cost and results in the formation of fine particles (17-19). However, physical stability of nanosuspensions prepared by this method can be impaired due to rapid unidirectional growth forming needle-shaped crystals (20).

Tadalafil is a phosphodiesterase 5 inhibitor used orally to treat male erectile dysfunction and marketed under the brand name Cialis ${ }^{\circledR}$. It has the advantage of long duration of action (21); however, it suffers poor aqueous solubility (22) leading to variability in blood levels and irreproducibility in clinical response. 
The aim of this work was to prepare, evaluate and stabilize nanosuspension formulation of Tadalafil via screening various types of stabilizers the anti-solvent precipitation method along with the advanced technology of ultrasonication. Solid dispersion of Tadalafil in PEG 4000 and its effect on enhancing dissolution of Tadalafil were also investigated and compared with the nanosuspension formulation.

\section{MATERIALS AND METHODS}

\section{Materials}

Tadalafil, sodium (Na) lauryl sulphate (SLS), polyvinylpyrrolidone (PVP) K-30, Pluronic F-127, Methocel E50, Methocel E5 LV, Cremophor EL and Brij58 were all generously gifted from TQ Pharma (Amman, Jordan); Span20, Span60 and Span80, PEG 4000 were purchased from (Fluka Chemie, Buchs, Switzerland); Tween80 was obtained from (JHD ${ }^{\mathrm{TM}}$, Guangdong, China); Triton X-100 from (Fisher Scientific UK Ltd, England, UK); sodium docusate was obtained from (Acros Organics, Morris, NJ, USA); magnesium stearate was obtained from (Fizmerk India Chemicals, India) and talc was obtained from (Riedel-De Haenag, Seelze-Hannover, Germany). All materials were used as received.

\section{Instruments}

The instruments were probe ultrasonicator (VibraCell ${ }^{\mathrm{TM}}$, VC 505, Sonics \& Materials, Inc., Newtown, CT, USA), SCHOTT pH meter (CG 843, Germany), Carver Press (Wabash, IN, USA), Malvern Zetasizer (ZS) (Malvern Instruments, Malvern, UK), Microtrac S3500 (USA), lyophilizer (Telstar, LyoQuest-85, Spain), differential scanning calorimeter (DSC-50 Shimadzu, Japan), Fourier transform infrared (FT-IR) spectrophotometer (IRAffinity-1, Shimadzu, Japan), UV-visible spectrophotometer CE (Cintra 5, GBC Scientific Equipment, Bausch \& Lomb, Rochester, NY, USA) and USP dissolution apparatus II (Copley Scientific, NE4-COP, UK).

\section{Preparations of Tadalafil Nanosuspension and Screening for Stabilizers}

In this study, an organic solution of Tadalafil was added to an aqueous solution containing a stabilizer, under sonication. Acetone and water were used as a solvent and an anti-solvent, respectively, at ratios that ranged from 1:49 to 1:15.6. Briefly, a weighed amount of 10,30 or $50 \mathrm{mg}$ of Tadalafil were completely dissolved in sufficient volume of acetone to prepare an organic solution of the drug which was then injected into specific volume of aqueous solution containing a stabilizer or mixture of them at different concentrations (in percent, weight/volume) at $5^{\circ} \mathrm{C}$ under stirred conditions and continuous ultrasonication. Stabilizers used included SLS, PVP K-30, Pluronic F-68, Tween80, Methocel E50, Methocel E5 LV, Triton X-100, Docusate sodium, Cremophor EL, Brij58, Span20, Span60 and Span80, in addition to combinations of some of them at different ratios (in percent, weight/volume). Continuous sonication was applied via a probe ultrasonicator for different time intervals. The prepared nanosuspensions were stirred at room temperature for $24 \mathrm{~h}$ to remove the organic solvent. Results we compared to a control preparation containing the drug probe-sonicated without any stabilizer.

\section{Effect of pH on Stability of Surfactant-Stabilized Nanosuspensions}

Nanosuspensions containing different stabilizers at different concentrations were prepared in acidic medium of $\mathrm{pH} 3$ and in alkaline medium of $\mathrm{pH} 10$, and results of particle size analysis and nanosuspension stability were compared to those prepared in neutral distilled water $(\mathrm{pH} 6.5)$.

\section{Effect of Organic: Aqueous Phase Ratio and Effect of Organic Phase Injection Rate}

The effects of the ratio of organic to aqueous phase and organic phase injection rate on Tadalafil nanosuspensions mean particle sizes prepared using the optimal ratio of Tween 80 and Span80 in neutral $(\mathrm{pH}$ 6.5) or in alkaline ( $\mathrm{pH} 10)$ media were investigated.

\section{Preparations of PEG-Tadalafil Matrices}

PEG 4000 was dissolved in freshly prepared Tadalafil nanosuspension or in Tadalafil raw powder suspensions in water and stirred for $24 \mathrm{~h}$. The mixture was then dried either using hot oven at $40^{\circ} \mathrm{C}$ or via freeze dry. The dried powder products (approximately $300 \pm 5 \mathrm{mg}$ ) were manually mixed for 10 min with $\mathrm{Mg}$ stearate and talc (at $0.25 \%$ each) and directly compressed via Carver Press at 1,000 lb for $30 \mathrm{~s}$. Additionally, a physical mixture of Tadalafil raw powder and PEG 4000 was also mixed with $\mathrm{Mg}$ stearate and talc for $10 \mathrm{~min}$ and then was

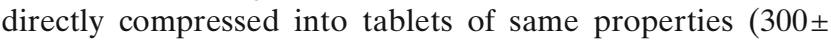
$5 \mathrm{mg}$, at 1,000 $\mathrm{lb}$ for $30 \mathrm{~s}$ ). Each tablet contained about $20 \mathrm{mg}$ of Tadalafil. The tablets were produced at room temperature between 23 and $26^{\circ} \mathrm{C}$ with the humidity between 37 and $42 \% \mathrm{RH}$ and were stored in airtight containers in the same room of preparation for further study. Tablets were characterized in terms of crushing strengths, interactions of components and dissolution properties.

\section{Particle Size Characterization}

Nanosuspensions were characterized in terms of mean particle size using dynamic light scattering, also known as photon correlation spectroscopy, using a Malvern ZS. Larger particles $(>1 \mu)$ were measured using a Microtrac S3500. Prior to the measurement, all samples were diluted using ultrapurified water to yield a suitable scattering intensity. Samples were analysed at $25^{\circ} \mathrm{C}$ using the general purpose mode.

\section{Freeze Dry}

Tadalafil nanosuspensions and Tadalafil raw powder suspensions containing PEG 4000 were lyophilized using trays that were frozen in deep freezer at $-80^{\circ} \mathrm{C}$ for $24 \mathrm{~h}$ for freezing. The trays were then transferred to the vacuum adapter of lyophilizer. The solvent was sublimed under a pressure of $0.01 \mathrm{mbar}$ for $48 \mathrm{~h}$. 


\section{Differential Scanning Calorimetry Analysis}

Differential scanning calorimetry (DSC) scans of pure Tadalafil powder, PEG 4000 and formulations A, B, C, D and $\mathrm{E}$ were recorded. Samples of approximately 1-7 mg were heated to $350^{\circ} \mathrm{C}$ at $10^{\circ} \mathrm{C} / \mathrm{min}$. Pierced aluminium pans were used for all samples. Pure indium was used to calibrate the DSC temperature scale.

\section{FT-IR Spectroscopic Analysis}

FT-IR spectra of moisture-free pure Tadalafil powder, PEG 4000 and formulations A, B, C, D and E powders were obtained using a FT-IR spectrophotometer using $\mathrm{KBr}$ as a reference. The scanning range was $450-4,000 \mathrm{~cm}^{-1}$.

\section{Drug Analysis}

The analysis for Tadalafil was carried out using the UV/ VIS spectrophotometer at the $\lambda_{\max }$ of the drug which is $285 \mathrm{~nm}$ using a quartz cuvette cell and against an appropriate blank. A standard solution composed of $20 \mathrm{ng} / \mathrm{ml}$ Tadalafil in appropriate solvent (distilled water containing $0.3 \%$ SLS at $\mathrm{pH} 6.5 \pm 0.5$ ) was prepared and serially diluted into a range of concentrations from 2 to $16 \mathrm{ng} / \mathrm{ml}$ whose absorbances were measured. This procedure was repeated three times, and an average of absorbances was taken at each point and plotted against the corresponding concentration to determine the linearity by calculating the correlation coefficient $\left(r^{2}\right)$ using linear regression analysis. UV scanning was performed on standard solutions of Tadalafil $(2 \mathrm{ng} / \mathrm{ml})$ dissolved in distilled water containing $0.3 \%$ SLS at $\mathrm{pH} 6.5 \pm 0.5$ and on Tadalafil solution $(2 \mathrm{ng} / \mathrm{ml})$ containing $0.05 \%$ Span 80 and $0.05 \%$ Tween 80 at $\mathrm{pH} 6.5 \pm 0.5$ or $\mathrm{pH} 10 \pm 0.5$.

\section{In Vitro Dissolution Studies}

Dissolution studies were performed for $20 \mathrm{mg}$ of plain Tadalafil raw powder and for an equivalent amount of Tadalafil nanosuspension ( $36 \mathrm{ml}$ Tadalafil nanosuspension of $50 \mathrm{mg} / 90 \mathrm{ml}$ strength, stabilized by Span 80 and Tween 80 at a $1: 1$ ratio (in percent, weight/volume) and $0.10 \%(w / v)$ total concentration). Dissolution was performed in 1,000 $\mathrm{ml}$ aqueous solution containing $0.3 \%(w / v)$ SLS and in $1,000 \mathrm{ml}$ aqueous solution containing Span80 and Tween80 at 1:1 ratio (in percent, weight/volume) and $0.10 \%(w / v)$ total concentration using USP dissolution apparatus II at $50 \mathrm{rpm}$ and $37 \pm 0.5^{\circ} \mathrm{C}$. Aliquots were periodically withdrawn $(5 \mathrm{ml})$ and were analysed spectrophotometrically at $285 \mathrm{~nm}$ and were replaced with an equal volume of dissolution medium. Samples were tested in triplicates, and an average of these was calculated and plotted against dissolution time.

\section{RESULTS AND DISCUSSION}

The ability to form nanosuspensions of poorly soluble drugs and maintaining the thermodynamic stability of suspended drug particles is quite challenging for some compounds. Thermodynamic stability of a suspension demands that the energy of interaction between solid dispersed drug particles to be of a repulsive type. In addition, adequate wetting of these particles by the liquid vehicle is required (23). The relevant time scales of dispersion stability may vary from tenths of seconds to years, depending on the application (24). In this study, an attempt was made to prepare nanosuspension of Tadalafil by the anti-solvent precipitation method using probe ultrasonication technique with the aid of stabilizers. According to Kocbek et al. (25), a drug which is a suitable candidate for nanosuspension preparation by this method should be poorly soluble in water (solubility of $<10^{-3}$ to $\left.10^{-4} \mathrm{~mol} / \mathrm{l}\right)$, but soluble in the selected organic solvent. Tadalafil with an aqueous solubility of $2 \mu \mathrm{g} / \mathrm{ml}$ (or $\left.5.3 \times 10^{-6} \mathrm{~mol} / \mathrm{l}\right)(22)$ possesses a significantly better solubility in organic solvents such as acetone which is miscible with water. However, Tadalafil is slightly soluble in acetone and some other organic solvents such as methanol and ethanol (26) which limits the amount of drug to be loaded to form concentrated nanosuspensions. A Tadalafil preparation probesonicated without any stabilizer being added to the aqueous phase resulted in the formation of a suspension containing large particles (hundreds of microns in size), indicating the need of certain molecules to stabilize the newly created surfaces formed upon sonication.

\section{Formulation of Nanosuspensions Using Single Stabilizers}

Polymers can be used to stabilize dispersions with the type of stabilization mechanism being steric in nature, particularly for uncharged polymers $(27,28)$. It is reported that polymers can be effective stabilizing agents only if the polymer-solvent interactions are more favourable than the polymer-polymer interactions (29). In this study, polymers such as PVP-K30, hydroxypropyl methylcellulose (Methocel E5 LV) and polyoxyethylene-polyoxypropylene block copolymers (Pluronic F-68) were employed to stabilize Tadalafil nanosuspensions at concentration shown in Table I (formulations 1-6), where results and observations are also shown. PVP-K30 containing formulation resulted in fluffy precipitate few minutes after sonication at different sonication times. Pluronic F-68 resulted in the formation of large particles that remained suspended for few hours. Low concentration of Methocel E5 LV yielded clear solutions with no visually apparent particles; however, 3 days after preparation, particle size measurement using Malvern nanosizer indicated the presence of particles with an average size of $11-17 \mu \mathrm{m}$ depending on duration of sonication. Higher concentrations of Methocel E5 LV produced only smaller particle size within the micrometer size range. On further storage, particle sizes increased forming a precipitate that was easily re-dispersed in the viscous environment. Although Methocel E5 LV produced the best results among the polymers employed, it was insufficient to prepare and stabilize Tadalafil nanosuspensions.

These results indicate that these polymers, with exception of Methocel E5 LV, were unable to be adsorbed efficiently and/or properly on the nonpolar Tadalafil particle surfaces in a way that provided steric hindrance. PVP-K30 relies on large dipole moment of the polymer side groups that interact with any other dipole present in the system. However, for Tadalafil, it seems that this dipole-dipole interaction (van der Waal forces) was insufficient to form a complex with PVP since Tadalafil possesses a relatively small polar surface area of $74.87 \AA^{2}$ (when compared to other poorly soluble drugs such 
Table I. Mean Particle Size (in Micrometres) and Macroscopic Appearance of Tadalafil Nanosuspensions (pH 6.5) Containing 10 mg Tadalafil Dissolved in $2 \mathrm{ml}$ Acetone and Stabilized with Polymers or Surfactant(s) Dissolved in $98 \mathrm{ml}$ Deionized Distilled Water (pH 6.5) Immediately After Sonication for $3 \mathrm{~min}$ at $50 \%$ of the Maximum Sonication Intensity at $5^{\circ} \mathrm{C}$

\begin{tabular}{|c|c|c|c|c|}
\hline $\begin{array}{l}\text { Property } \\
\text { Tadalafil } \\
\text { formulation }\end{array}$ & Stabilizer (s) & Concentration $(\%, w / v)$ & Mean particle size $(\mu \mathrm{m})$ & Macroscopic appearance \\
\hline 1 & PVP K30 & 0.50 & $33.7 \pm 17.2$ & Fluffy precipitate within few minutes \\
\hline 2 & & 1.0 & $31.9 \pm 14.3$ & Fluffy precipitate within few minutes \\
\hline 3 & & 1.50 & $10.5 \pm 5.6$ & Fluffy precipitate within few minutes \\
\hline 4 & Methocel E5 LV & 0.15 & $10.1 \pm 3.8$ & Clear solution for $24 \mathrm{~h}$ \\
\hline 5 & & 0.50 & $4.5 \pm 1.8$ & Clear solution for $24 \mathrm{~h}$ \\
\hline 6 & Pluronic F-68 & 0.10 & $153 \pm 32.8$ & Particles agglomerated and precipitated \\
\hline 7 & SLS & 0.05 & $52.8 \pm 16.8$ & Immediate agglomeration \\
\hline 8 & & 0.10 & $33.6 \pm 10.2$ & Immediate agglomeration \\
\hline 9 & Na docusate & 0.05 & $103 \pm 29.4$ & Immediate agglomeration \\
\hline 10 & & 0.10 & $98 \pm 22.6$ & Immediate agglomeration \\
\hline 11 & & 0.50 & $113 \pm 22.2$ & Immediate agglomeration \\
\hline 12 & Triton X-100 & 0.5 & $33.7 \pm 14.2$ & Clear solution with thread-like particles \\
\hline 13 & & 1.0 & $31.9 \pm 11.6$ & Clear solution with thread-like particles \\
\hline 14 & & 1.5 & $10.5 \pm 2.7$ & Clear solution with thread-like particles \\
\hline 15 & Brij58 & 0.05 & $0.237 \pm 0.1$ & Colloidal solution for $3 \mathrm{~h}$ \\
\hline 16 & & 0.2 & $2.497 \pm 0.6$ & Colloidal dispersion for $3 \mathrm{~h}$ \\
\hline 17 & & 0.5 & $119 \pm 28.8$ & Clear thread-like particles \\
\hline 18 & Cremophor EL & 0.05 & $19.02 \pm 0.5$ & Colloidal dispersion for $3 \mathrm{~h}$ \\
\hline 19 & & 0.10 & $25.6 \pm 6.9$ & Colloidal dispersion for $3 \mathrm{~h}$ \\
\hline 20 & & 0.2 & $9.4 \pm 0.8$ & Colloidal dispersion for $3 \mathrm{~h}$ \\
\hline 21 & & 0.50 & $1.38 \pm 0.4$ & Colloidal dispersion for $3 \mathrm{~h}$ \\
\hline 22 & & 1.0 & $40.2 \pm 6.8$ & Clear solution with thread-like particles \\
\hline 23 & Tween80 & 0.0025 & $20.8 \pm 5.7$ & Colloidal dispersion for $24 \mathrm{~h}$ \\
\hline 24 & & 0.01 & $1.29 \pm 0.3$ & Colloidal dispersion for $24 \mathrm{~h}$ \\
\hline 25 & & 0.05 & $0.75 \pm 0.1$ & Colloidal dispersion for $72 \mathrm{~h}$ \\
\hline 26 & & 0.10 & $0.788 \pm 0.1$ & Colloidal dispersion for $72 \mathrm{~h}$ \\
\hline 27 & & 0.20 & $1.4 \pm 0.4$ & Colloidal dispersion for $3 \mathrm{~h}$ \\
\hline 28 & & 0.50 & $4.7 \pm 1.7$ & Colloidal dispersion for $3 \mathrm{~h}$ \\
\hline 29 & Tween80/SLS & $0.05: 0.10$ & $129 \pm 10.2$ & Agglomeration within $1 \mathrm{~h}$ \\
\hline 30 & & $0.10: 0.20$ & $5 \pm 0.6$ & Agglomeration within $1 \mathrm{~h}$ \\
\hline 31 & Tween $80 / \mathrm{Na}$ docusate & 0.05:0.01 & $0.46 \pm 0.1$ & Agglomeration and precipitation within $1 \mathrm{~h}$ \\
\hline 32 & & 0.05:0.05 & $0.50 \pm 0.1$ & Agglomeration and precipitation within $1 \mathrm{~h}$ \\
\hline 33 & Brij58/Span 80 & $0.20 / 0.05$ & $1.7 \pm 0.4$ & Agglomeration within $24 \mathrm{~h}$ \\
\hline 34 & Cremophor EL/Span 80 & $0.20 / 0.05$ & $2.8 \pm 1.1$ & Colloidal dispersion for $1 \mathrm{~h}$ \\
\hline 35 & Tween80/Cremophor EL & 0.10:0.10 & $19.7 \pm 4.4$ & Precipitation within $3 \mathrm{~h}$ \\
\hline 36 & & $0.5: 0.5$ & $74.50 \pm 18.2$ & Precipitation within $3 \mathrm{~h}$ \\
\hline 37 & Tween80/Brij58 & 0.05:0.05 & $10.61 \pm 0.1$ & Colloidal dispersion for $3 \mathrm{~h}$ \\
\hline 38 & Tween80/Span20 & 0.10:0.10 & $1.26 \pm 0.2$ & Colloidal dispersion for $3 \mathrm{~h}$ \\
\hline 39 & Tween $80 /$ Span60 & $0.20: 0.20$ & $99.14 \pm 30.1$ & Colloidal dispersion for $1 \mathrm{~h}$ \\
\hline 40 & Tween80/Span 80 & $0.05: 0.05$ & $0.205 \pm 0.1$ & Colloidal dispersion for $72 \mathrm{~h}$ \\
\hline
\end{tabular}

PVP polyvinylpyrrolidone, $S L S$ sodium lauryl sulphate

as glibenclamide with polar surface area of $121.98 \AA^{2}$ ) (30). Results were supported by the observation that the particles stabilized using PVP-K30 were fluffy and similar in appearance to stabilizer-free Tadalafil nanosuspensions.

\section{Formulation of Nanosuspensions Using Single Surfactants}

Surfactants are known to interfere with the interfacial transport of solute from the crystal to the bulk solution (31). The solubility enhancement properties of surfactants resulted from the dual nature of surfactant molecules (32).

Ionic surfactants were theoretically claimed to aid in preparation and stabilization Tadalafil nanosuspensions; however, results of this study did not support the claim. Both SLS and $\mathrm{Na}$ docusate were unable to stabilize the newly formed particles when employed at different concentrations in neutral medium ( $\mathrm{pH}$ of 6.5 ) as shown in Table I (formulations 7-11). SLS-containing formulations resulted in an immediate formation of Tadalafil particles with size range of $38-71 \mu \mathrm{m}$ depending on its concentration and duration of sonication. Similar results were observed for Na docusate containing formulations which yielded particles in the size range of 90-98 $\mu \mathrm{m}$ under the same conditions. These results indicated that both surfactants (SLS and $\mathrm{Na}$ docusate) were unable to be adsorbed sufficiently and/or properly onto Tadalafil particle surfaces. This could be due to high solubility and insufficient hydrophobicity of lauryl chain of SLS and the inappropriate orientation of the side succinate chains of $\mathrm{Na}$ docusate on particle surfaces.

Non-ionic surfactant molecules offer an advantage over polymers in that they are amphiphilic surfactant molecules 
and typically have a higher adsorption potential than an equal chain length polymer. Results of non-ionic surfactants employed individually for the preparation of Tadalafil nanosuspensions at $\mathrm{pH}$ of 6.5 are shown in Table I (formulations 12-28). Brij58-stabilized nanosuspensions at $0.05 \%(w / v)$ were colloidal with an average particle size of about $237 \mathrm{~nm}$ at low sonication times; however, short time after storage (within less than $3 \mathrm{~h}$ ) particles exhibited a marked growth. At $0.20 \%(w / v)$, larger particles were resulted (about $2.5 \mu \mathrm{m}$ ) with agglomerations that appeared within $24 \mathrm{~h}$. At higher concentrations $(0.5 \%$, $w / v)$, clear transparent solutions with thread-like structures having an average size of $119 \mu \mathrm{m}$ were observed.

Using Cremophor EL as a stabilizer at concentrations of $0.02 \%(w / v)$ and $0.05 \%(w / v)$ resulted in particles in the size range of $1.2-4.5 \mu \mathrm{m}$, depending on sonication time. At $0.10 \%$, particles aggregation occurred. At $0.5 \%$, dispersions were colloidal in appearance but with particle size range of 1.4$9.85 \mu \mathrm{m}$. Higher concentrations of Cremophor EL $(0.75 \%$ and $1 \%)$ showed clear transparent solutions with thread-like particles in the size range of 10-97 $\mu \mathrm{m}$, which were similar in appearances to dispersions stabilized with Triton X-100 at concentrations of $0.50,1.0$ and $1.50 \%$, where particles displayed sized ranging from 7.0 to $71.5 \mu \mathrm{m}$.

At $0.0025 \%(w / v)$ (close to its CMC value), Tween 80 aided in the formation of colloidal dispersion with an average particle size range of 1.0-20.7 $\mu \mathrm{m}$ at both long and short sonication times. At concentration ten times its CMC value, Tween 80 yielded colloidal dispersions with an average particle size being close to $1 \mu \mathrm{m}$; however, precipitation occurred 1 day after preparation. At $0.05 \%(w / v)$ and $0.10 \%$, colloidal dispersions with an average particle size of $750 \pm 15$ and $788 \pm$ $22 \mathrm{~nm}$, respectively, were produced in distilled water ( $\mathrm{pH} 6.5$ ). Both preparations experienced certain magnitude of stability where further increase in particle size was noticed only 3 days after production.

\section{Formulation of Nanosuspensions Using Combinations of Surfactants}

The use of certain sole surfactants (e.g. Tween80) at specific concentrations produced a reduction in particle size of Tadalafil suspension to the nanometre size range. However, further reduction in the average nanosuspension particle size was needed. Also, nanosuspensions produced were unstable enough to allow further processing. This could be due to the inability of adsorbed surfactant molecules to form a complex, well-packed and flexible film with sufficient electrostatic and/ or steric characteristics on the surfaces of Tadalafil particles. Therefore, combinations of surfactants were employed in the next step to prepare nanosuspensions with sufficient stability to permit further handling procedures. The rationale behind using mixtures of surfactants is coupled with the ability of certain mixtures to behave synergistically for stabilization purposes, particularly in cases where single surfactant does not perform well. This synergistic behaviour can be typically in the form mixed aggregates or structures, as in mixed monolayers and mixed bilayers with a more fluid-like or solid-like behaviour than either of their single surfactant aggregates. Often the synergistic effect of the mixed systems has been shown to be due to enhanced adsorption from the mixture of a weakly adsorbing component (33). Combinations of surfactants employed in current study are shown in Table I (formulations 29-40) along with their results in neutral medium ( $\mathrm{pH} 6.5)$. Combinations of two surfactants were added at several ratios with total concentrations well above the CMC of any of the surfactants. When combinations of Tween 80 and SLS or Tween 80 and $\mathrm{Na}$ docusate were used as stabilizer mixtures, suspensions had average particle sizes of about 129 and $0.5 \mu \mathrm{m}$, respectively, at the highest concentrations employed of both combinations. Both formulations were unstable and showed particle growth and agglomerations shortly after preparation (within $3 \mathrm{~h}$ ) indicating that the aforementioned surfactant combinations were apparently unable to form such a film that prevent particles from coming close to each other towards agglomeratation. Therefore, the adsorption that might have happened initially (as in case of individual Tween 80 or $\mathrm{Na}$ docusate) was followed by agglomeration and precipitation.

Combinations of nonionic surfactants such as a mixture of Tween80 and Cremophor EL resulted in large particle size suspensions (19.72-74.5 $\mu \mathrm{m}$ ) depending on total concentration used. The size range obtained using this combination was similar to that obtained using Cremophor EL alone as a stabilizer indicating that Tween 80 did not interfere with Cremophor EL adsorption process. Mixtures of Tween80/Brij58, Brij58/Span80 and Cremophor EL/Span80 experienced insignificant reduction in the average particle size compared to single surfactants, indicating insignificant synergism.

Mixtures of Tween 80 and Spans 20,60 or 80 were used to stabilize nanosuspensions at $1: 1$ ratio and $0.1 \%$ total concentration. Span20 and Span60 are identical in their structures, except for the side chain lengths. Despite that mixtures of Tween 80 with Span20 or Span60 resulted in particles in nanometre size range, particles aggregated within hours in neutral media (pH 6.5). Comparatively, when Span 80 was combined with Tween 80 at the same conditions, nanosuspension produced possessed an average size range of $(205 \pm 6 \mathrm{~nm})$ with an observed stability for 3 days (almost constant mean particle size during this period). Nanosuspensions were colloidal in appearance and were free of black particles associated with the use of either Span20 Span60. However, the increase in particle size after 3 days can be attributed to the low zeta potential $(-8.71 \mu \mathrm{S})$ of Tadalafil nanoparticles. Figure 1 shows the chemical structures of Span 80 and Tween 80 where it is

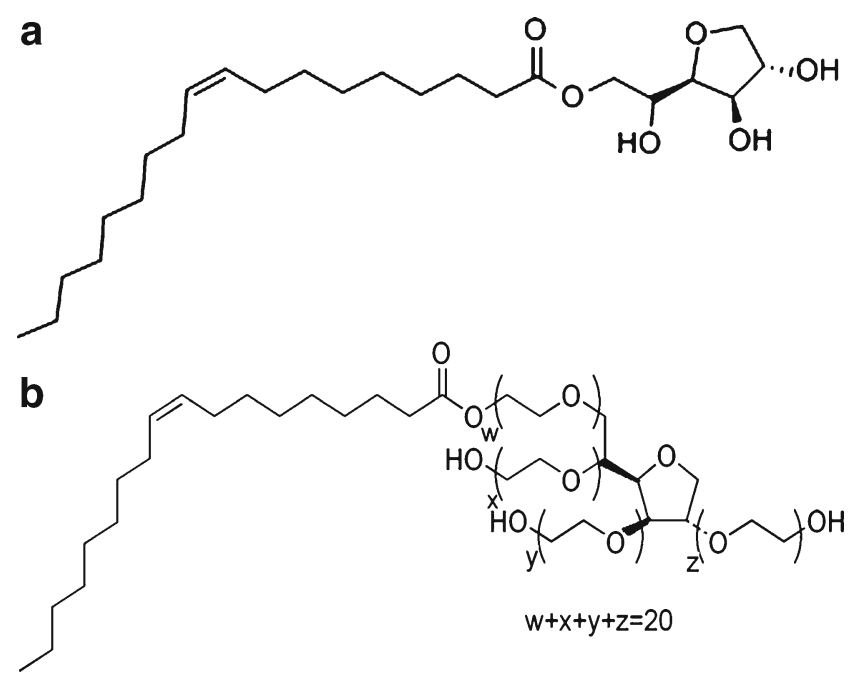

Fig. 1. Chemical structures of a Span 80 and b Tween 80 

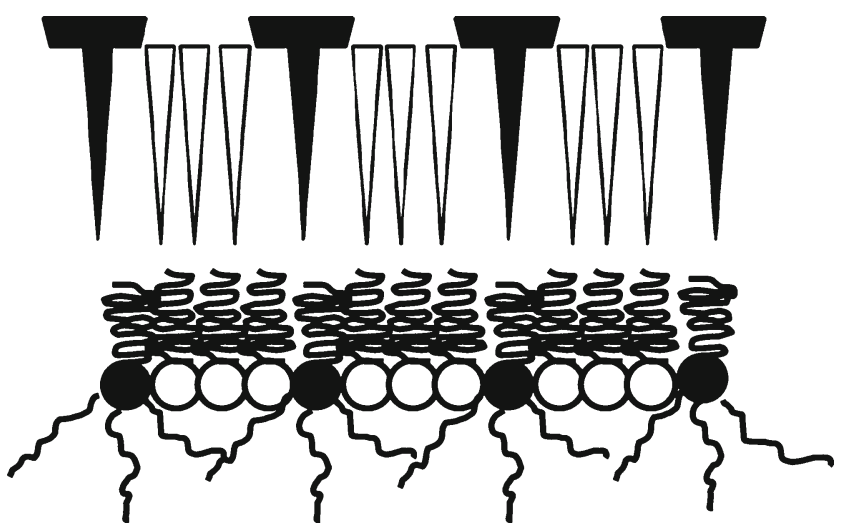

Fig. 2. Interaction models of surfactants (white triangles and circles) Span80 and (black triangles and circles) Tween 80

apparent that Span80 is similar in structure to Spans20 and 60 except for its chain length (16 carbons in Span80, 12 and 18 in Span20 and Span60, respectively) and its nature (unsaturated in Span80).

The achievement of nanosized particles of a relatively adequate stability can be explained by the formation of a mixed monolayer conformation in which the hydrocarbon chains of the two surfactants (Tween80 and Span80) are lined up adjacent to each other (34). This type of conformation is favoured if the amount of adsorbed Span80 is low enough creating gaps between molecules that are large enough for the Tween 80 hydrocarbon chains to fully penetrate, as shown in Fig. 2. In the depicted model, it can be noticed that Span 80 could act as a barrier of steric interactions between polar groups on Tween 80 preventing their close proximity. The mixed monolayer conformation will result in the lowest surfactant adsorption density of any of the conformations possible in this mechanism. Other possibilities of conformation can exist, for example, a full bilayer conformation which is favoured when there is enough Tween 80 in solution to adsorb as a full second monolayer (35). However, the first conformation seemed to be more appropriate in the current case since nanosuspension dispersion stability and particle size were found to be worse at higher total surfactant concentrations $(\geq 0.20 \%, w / v)$. At higher surfactant concentrations, adsorption of a third surfactant layer of Span 80 or Tween 80 could occur, or alternatively the solubility limit of Span 80 in water could be reached causing precipitation.

Span80 has greater hydrophobicity $(\mathrm{HLB}=4.3)$ than either Span20 $(\mathrm{HLB}=8.6)$ or Span60 $(\mathrm{HLB}=4.7)$, and thus, it was expected to have more affinity for Tadalafil hydrophobic surfaces. Tween 80 is a hydrophilic surfactant and was shown to exhibit great steric stabilization when used alone (nanosuspensions size range of $750 \pm 15 \mathrm{~nm}$ ) in neutral medium. However, the synergistic action of Tween 80 and Span80 combination was found to bring the nanosuspension particle size to about $205 \pm 18 \mathrm{~nm}$ with a relatively adequate stability.

The optimum ratio of Tween 80 and Span 80 combination was found to be 1:1 ratio (in percent, weight/volume) at a total concentration of $0.10 \%(w / v)$. The calculated $\mathrm{HLB}_{\text {mix }}$ of the mixture was 9.65. On the other hand, the molar ratio of Span80/Tween80 was close to 3:1. Other ratios (in percent, weight/volume) of Span80/Tween80 at a fixed total concentration of $0.10 \%(w / v)$ were also investigated in order to determine the effects of $\mathrm{HLB}_{\text {mix }}$ and molar ratios of surfactants on nanosuspension properties. Both at lower molar ratio of Span80/Tween80 of 1.3:1 $\left(\mathrm{HLB}_{\mathrm{mix}}=11.8\right)$ and at higher ratios of $2: 1 \quad\left(\mathrm{HLB}_{\mathrm{mix}}=\right.$ 10.72) and 6:1 $\left(\right.$ HLB $\left._{\mathrm{mix}}=7\right)$, nanosuspensions of large particles resulted and were readily unstable. Therefore, an

Table II. Mean Particle Size (in Nanometres) for Tadalafil Nanosuspension Containing $10 \mathrm{mg}$ Tadalafil Dissolved in 2 ml Acetone and Stabilized Using Single or Mixtures of Surfactants of Different Concentrations Dissolved in $98 \mathrm{ml}$ Deionized Distilled Water of Different pH Values (3, 6.5 and 10) Immediately After Sonication for $3 \mathrm{~min}$ at $50 \%$ of the Maximum Sonication Intensity at $5^{\circ} \mathrm{C}$

\begin{tabular}{|c|c|c|c|c|c|}
\hline \multirow{2}{*}{$\begin{array}{l}\text { Property } \\
\text { Tadalafil formulation }\end{array}$} & \multirow[t]{2}{*}{ Stabilizer type } & \multirow[t]{2}{*}{ Stabilizer concentration $(\%, w / v)$} & \multicolumn{3}{|c|}{ Mean particle size (nm) } \\
\hline & & & $\mathrm{pH} 3$ & pH 6.5 & $\mathrm{pH} 10$ \\
\hline 1 & Tween80 & 0.05 & $609 \pm 16.9$ & $750 \pm 15.8$ & $202 \pm 4.5$ \\
\hline 2 & Tween80 & 0.10 & $208 \pm 8.9$ & $788 \pm 22.3$ & $145 \pm 4.3$ \\
\hline 3 & Tween80 & 0.20 & $740 \pm 20.1$ & $1,400 \pm 42.5$ & $555 \pm 18.3$ \\
\hline 4 & Brij58 & 0.05 & $394 \pm 8.5$ & $237 \pm 10.8$ & $486 \pm 28$ \\
\hline 5 & Brij58 & 0.20 & $1,140 \pm 120$ & $2,497 \pm 299$ & $1,050 \pm 149$ \\
\hline 6 & Cremophor EL & 0.05 & $379 \pm 6.8$ & $19,002 \pm 492.1$ & $352 \pm 9.1$ \\
\hline 7 & Cremophor EL & 0.20 & $393 \pm 10.2$ & $9,440 \pm 822$ & $138,600 \pm 12,035$ \\
\hline 8 & Tween80/SLS & 0.05:0.10 & $1,200 \pm 72.8$ & $129,000 \pm 1,022$ & $1,000 \pm 55.0$ \\
\hline 9 & Tween80/SLS & $0.10: 0.20$ & $40,300 \pm 4,123$ & $5,000 \pm 564$ & $73,920 \pm 6,768$ \\
\hline 10 & Tween80/Brij58 & $0.05: 0.05$ & $311 \pm 24$ & $1,061 \pm 123$ & $194 \pm 12$ \\
\hline 11 & Tween80/Brij58 & 0.10:0.10 & $754 \pm 37$ & $1,258 \pm 134$ & $1,583 \pm 126$ \\
\hline 12 & Tween80/Brij58 & $0.20: 0.20$ & $80,430 \pm 4,723$ & $99,140 \pm 6,234$ & $2,015 \pm 123.3$ \\
\hline 13 & Brij58/Span80 & $0.05: 0.05$ & $679 \pm 29$ & $475 \pm 16$ & $411 \pm 11$ \\
\hline 14 & Brij58/Span80 & $0.2: 0.05$ & $1,179 \pm 210$ & $1,660 \pm 144$ & $1,890 \pm 172$ \\
\hline 15 & Cremophor EL/Span 80 & $0.2: 0.05$ & $921 \pm 32$ & $2,793 \pm 312$ & $62,410 \pm 2,622$ \\
\hline 16 & Tween80/Span20 & $0.05: 0.05$ & $468 \pm 22.3$ & $208 \pm 9.8$ & $268 \pm 36.8$ \\
\hline 17 & Tween80/Span60 & $0.05: 0.05$ & $710 \pm 30$ & $542 \pm 33.7$ & $601 \pm 45.1$ \\
\hline 18 & Tween $80 / \operatorname{Span} 80$ & $0.05: 0.05$ & $316 \pm 13$ & $205 \pm 6$ & $193 \pm 8.0$ \\
\hline
\end{tabular}


Table III. Effect of the Ratio of Organic Phase to Aqueous Phase Ratio and Rate of Injection of the Organic Phase on the Mean Particle Size for Nanosuspension (in Nanometres) Stabilized in Alkaline or Neutral Media Using Tween80/Span80 at 1:1 Ratio (in Percent, Weight/Volume) and $0.10 \%(w / v)$ Total Concentration

\begin{tabular}{|c|c|c|c|c|c|}
\hline $\begin{array}{l}\text { Property } \\
\text { Tadalafil } \\
\text { formulations }\end{array}$ & Tadalafil raw material $(\mathrm{mg})$ & Organic solvent $(\mathrm{ml})$ & Flow rate $(\mathrm{ml} / \mathrm{min})$ & Aqueous phase $(\mathrm{ml})$ & Particle size (nm) \\
\hline TDTSp-OH-1 & 10 & 2 & 2 & 98 & $148 \pm 18$ \\
\hline TDTSp-OH-2 & 10 & 2 & 0.67 & 98 & $198 \pm 13$ \\
\hline TDTSp-OH-3 & 10 & 6 & 6 & 94 & $149 \pm 20$ \\
\hline TDTSp-OH-4 & 10 & 6 & 2 & 94 & $180 \pm 22$ \\
\hline TDTSp-OH-5 & 30 & 6 & 6 & 94 & $201 \pm 11$ \\
\hline TDTSp-OH-6 & 30 & 6 & 2 & 94 & $241 \pm 15$ \\
\hline TDTSp-OH-7 & 50 & 10 & 10 & 90 & $196 \pm 9$ \\
\hline TDTSp-OH-8 & 50 & 10 & 10 & 90 & $221 \pm 13$ \\
\hline TDTSp-N-1 & 10 & 6 & 6 & 94 & $185 \pm 10$ \\
\hline TDTSp-N-2 & 10 & 6 & 2 & 94 & $292 \pm 16$ \\
\hline TDTSp-N-3 & 10 & 10 & 10 & 90 & $401 \pm 33$ \\
\hline TDTSp-N-4 & 30 & 6 & 6 & 94 & $146 \pm 8$ \\
\hline TDTSp-N-5 & 30 & 6 & 2 & 94 & $193 \pm 15$ \\
\hline TDTSp-N-6 & 30 & 10 & 10 & 90 & $303 \pm 20$ \\
\hline TDTSp-N-7 & 50 & 10 & 10 & 90 & $197 \pm 7$ \\
\hline TDTSp-N-8 & 50 & 10 & 10 & 90 & $217 \pm 11$ \\
\hline
\end{tabular}

TDTSp-OH Tadalafil-Tween80-Span80 nanosuspension prepared in alkaline medium, TDTSp-N Tadalafil-Tween80-Span80 nanosuspension prepared in neutral medium

optimal ratio of both surfactants should be carefully chosen for formulating Tadalafil nanosuspensions.

\section{Effect of pH on Stability of Surfactant-Stabilized Nanosuspensions}

In an attempt to enhance the physical stability of Tadalafil nanosuspensions using surfactants as stabilizers, the effect of $\mathrm{pH}$ was investigated. Nanosuspensions were prepared in acidic medium of $\mathrm{pH} 3$ and in alkaline medium of $\mathrm{pH} 10$, and results were compared to those prepared in neutral distilled water ( $\mathrm{pH}$ 6.5) as shown in Table II (formulations 1-18). In general, the results indicated that, except for certain surfactants and surfactants combinations, the suspended particles exhibited a decrease in their mean sizes in the alkaline medi-

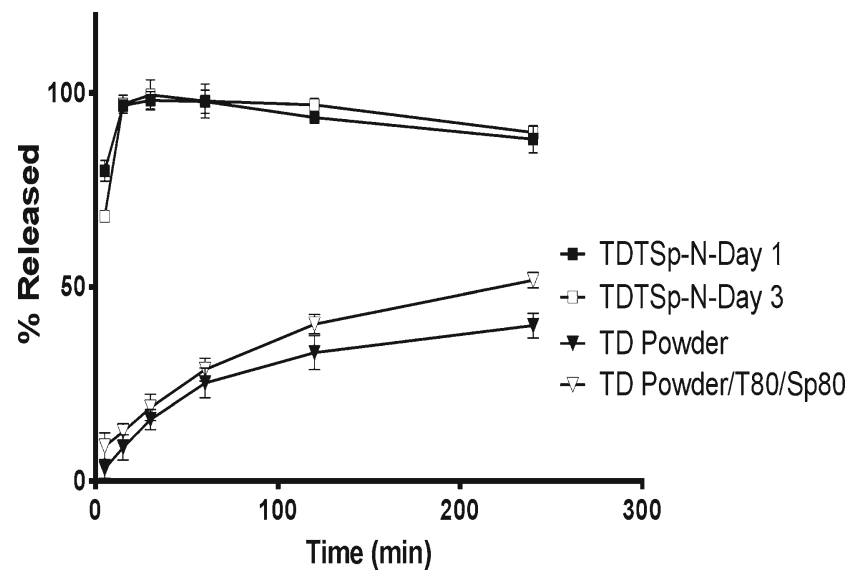

Fig. 3. Drug dissolution (release) for Tadalafil $(T D)$ raw material in $0.3 \%$ SLS and in $0.3 \%$ SLS $/ 0.05 \%$ Tween $/ 0.05 \%$ Span 80 and for Tadalafil nanosuspensions (TDTSp- $N$ ) in $0.3 \%$ SLS at $37^{\circ} \mathrm{C}$. All percentages are weight/volume um. Best stability was recorded for Tween80/Span80 in alkaline medium which showed stability up to 3 days with an almost constant mean particle size. Improved stability was attributed to adsorption of hydroxyl ions of nanosuspensions as indicated by the negative sign of zeta potential giving rise to certain degree of between dispersed particles.

\section{Effect of Organic: Aqueous Phase Ratio and Effect of Organic Phase Injection Rate}

Table III shows results of the effects of the ratio of organic to aqueous phase and organic phase injection rate on Tadalafil nanosuspensions mean particle sizes prepared using the optimal ratio of Tween 80 and Span 80 in neutral $(\mathrm{pH} 6.5)$ or in alkaline $(\mathrm{pH} 10)$ conditions. Results indicated that when the ratio of organic/aqueous phase was increased from 1:49 to

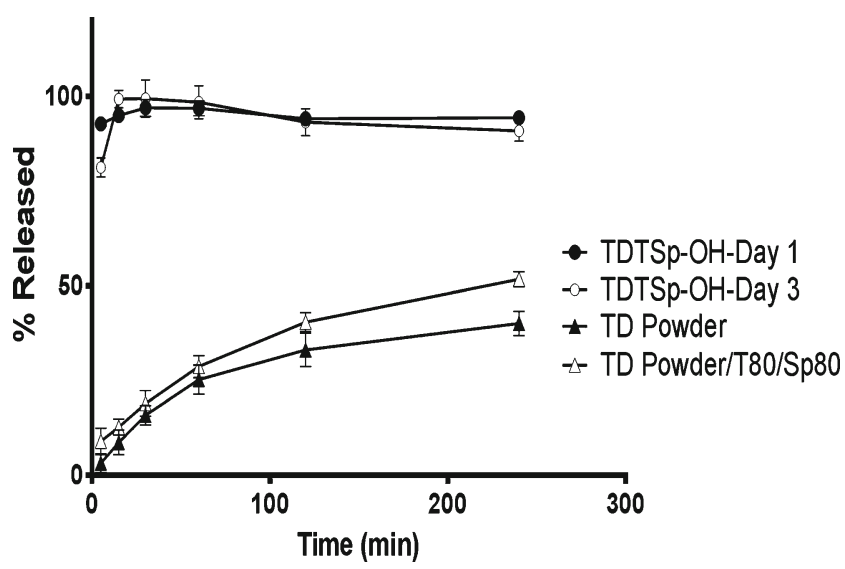

Fig. 4. Drug dissolution (release) for Tadalafil raw material (TD) in $0.3 \%$ SLS and in $0.3 \%$ SLS $/ 0.05 \%$ Tween $/ 0.05 \%$ Span 80 and for Tadalafil nanosuspensions (TDTSp-OH) in $0.3 \%$ SLS at $37^{\circ} \mathrm{C}$. All percentages are weight/volume 
Table IV. Composition and Drying Process of Tadalafil-PEG 4000 Solid Dispersion Matrices Directly Compressed via Carver Press at 1,000 lb for $30 \mathrm{~s}$

\begin{tabular}{|c|c|c|c|c|c|c|c|}
\hline $\begin{array}{l}\text { Property } \\
\text { Tablet } \\
\text { formulas }\end{array}$ & $\begin{array}{l}\text { TDTSp-OH }{ }^{a} \\
(\mathrm{ml})\end{array}$ & $\begin{array}{l}\text { Tadalafil/TSp-OH } \\
(\mathrm{mg})\end{array}$ & $\begin{array}{l}\text { PEG } \\
4000(\mathrm{mg})\end{array}$ & $\begin{array}{l}\text { Mg stearate } \\
(\mathrm{mg})\end{array}$ & Talc (mg) & $\begin{array}{l}\text { Tablet } \\
\text { weight (mg) }\end{array}$ & $\begin{array}{l}\text { Drying } \\
\text { process }\end{array}$ \\
\hline A & 36 & - & 280 & 0.75 & 0.75 & 300 & Oven, $40^{\circ} \mathrm{C}$ \\
\hline B & 36 & - & 280 & 0.75 & 0.75 & 300 & Freeze dry \\
\hline $\mathrm{C}$ & - & 20 & 280 & 0.75 & 0.75 & 300 & Oven, $40^{\circ} \mathrm{C}$ \\
\hline $\mathrm{D}$ & - & 20 & 280 & 0.75 & 0.75 & 300 & Freeze dry \\
\hline $\mathrm{E}$ & - & 20 & 280 & 0.75 & 0.75 & 300 & - \\
\hline
\end{tabular}

TDTSp-OH Tadalafil-Tween80-Span80 nanosuspension prepared in alkaline medium, TSp-OH Tween80-Span80 dispersed in alkaline medium ${ }^{a}$ Millilitres of TDTSp-OH Tadalafil nanosuspension (mean particle size $=193 \pm 8 \mathrm{~nm}$ and strength $=50 \mathrm{mg} / 90 \mathrm{ml}$ ) that is equivalent to $20 \mathrm{mg}$ Tadalafil

${ }^{b}$ Milligrams of Tadalafil raw powder (particle size $=10 \mu \mathrm{m}$ ) dispersed in TSp-OH

1:15.6, a significant increase in nanosuspensions particle sizes was noticed when all other parameters were kept constant (flow or injection rate, amount of drug, total volume, sonication time and intensity). The presence of the organic phase in a large proportion retained the drug as 'dissolved' shielding it from direct comminution via cavitation during sonication, and therefore, upon evaporation of the organic solvent, the dissolved drug will precipitate into larger particles. In addition, results have shown that the effect of the increase in organic to aqueous phase ratio was more pronounced at low injection rates. This can be attributed to the actual time span the whole precipitated drug particles spent during sonication. Thus, a relatively rapid drug injection would permit a bigger chance for the whole drug injected to be sonicated for longer times.

\section{In Vitro Dissolution of Optimized Tadalafil Nanosuspensions}

The dissolution profiles, 1 and 3 days after probe sonication of Tadalafil nanosuspensions containing $0.05 \%(w / v)$ Span 80 and $0.05 \%(w / v)$ Tween 80 at pH $6.5 \pm 0.5$ (TDTSp-N) or at $\mathrm{pH} 10 \pm 0.5$ (TDTSp-OH), were determined in $1,000 \mathrm{ml}$ of distilled water containing $0.3 \%(w / v)$ SLS. Three batches of TDTSp-N with mean particle sizes of 223, 232 and $244 \mathrm{~nm}$ and of TDTSp-OH with mean particle sizes of 215, 222 and $229 \mathrm{~nm}$ were tested, and the averages of these were compared to data obtained from the dissolution of raw powder of Tadalafil $(20 \mathrm{mg})$ in $1,000 \mathrm{ml}$ of distilled water containing $0.3 \%(\mathrm{w} / \mathrm{v})$ SLS or in $1,000 \mathrm{ml}$ of distilled water containing $0.3 \%(w / v)$ SLS and mixture of Span 80 and Tween 80 at $0.05 \%$ each. The method of analysis was validated with a correlation coefficient $\left(r^{2}\right)$ of the calibration curve of 0.9975 . The UV scans of Tadalafil/SLS solution and Tadalafil/SLS/surfactants $(0.05 \%$ $(w / v)$ Span 80 and $0.05 \%(w / v)$ Tween 80$)$ at neutral or alkaline conditions revealed that all Tadalafil solutions exhibited a maximum absorbance at about $285 \mathrm{~nm}$ and the absorbances of surfactants did not interfere with the assay of Tadalafil at this wavelength. Results of the dissolution profiles are shown in Figs. 3 and 4 after day 1 (1 day after probe sonication) and after day 3 , respectively. These figures clearly showed a dramatic increase in the rate and extent of dissolution for Tadalafil nanosuspensions in comparison with raw drug powder. This increase was more significant for TDTSp-OH nanosuspensions where more than $92 \%$ of Tadalafil was dissolved within the initial 5 min compared to about $80 \%$ for
TDTSp-N nanosuspensions and less than $10 \%$ for raw Tadalafil powder. In addition, it was apparent from figures that within the first 5 min of dissolution time, $80 \%$ and $70 \%$ of the cumulative drug release were observed for TDTSp-OH and TDTSp-N, respectively. However, drug dissolution was almost complete within the first $10 \mathrm{~min}$ for all nanosuspension formulations at both 1 and 3 days after preparation compared to less than $15 \%$ for raw Tadalafil powder.

\section{Drug Release from PEG-Tadalafil Directly Compressed Solid Dispersion Matrices}

In order to obtain Tadalafil nanosuspension into more stable and convenient dosage forms, Tadalafil nanosuspensions were transformed into tablet matrices using polyethylene glycol (PEG 4000) as a carrier. Five different formulations were prepared as shown in Table IV. In formulations A and B, PEG 4000 was dissolved in Tadalafil nanosuspensions (TDTSp-OH) immediately after sonication and stirred for $24 \mathrm{~h}$ until acetone was removed. Then the nanosuspension-PEG mixture was solidified either in oven at $40^{\circ} \mathrm{C}$ (formulation A) or via freeze dry (formulation B). In formulations C and D, PEG was dissolved in Tadalafil powder suspension containing Tween 80 and Span 80 with acetone being added to the suspension at concentrations mimicking that in TDTSp-OH and stirred for $24 \mathrm{~h}$ until acetone was evaporated. Then the powder suspension-PEG mixture

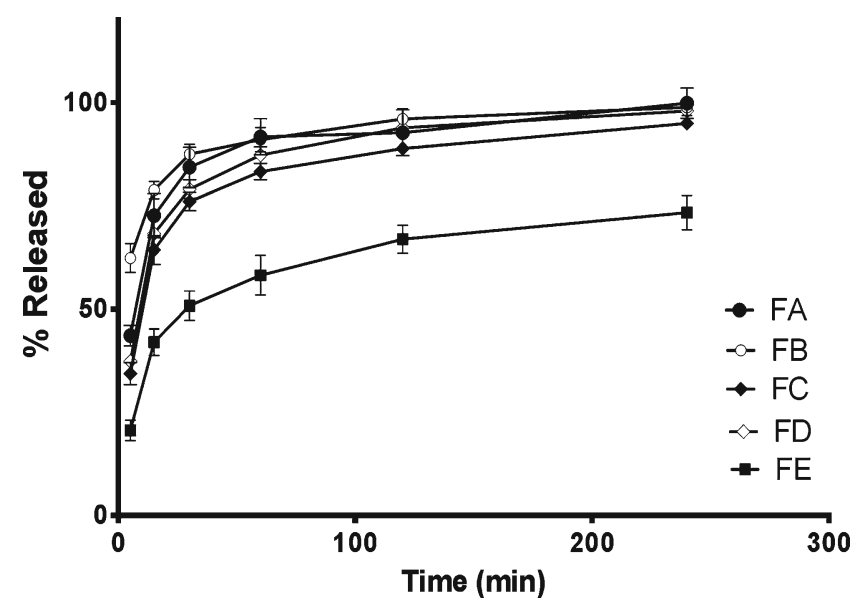

Fig. 5. Cumulative drug release for Tadalafil-PEG tablet formulations $A, B, C, D$ and $E$ in $0.3 \%(w / v) \mathrm{SLS}$ at $37^{\circ} \mathrm{C}$ 


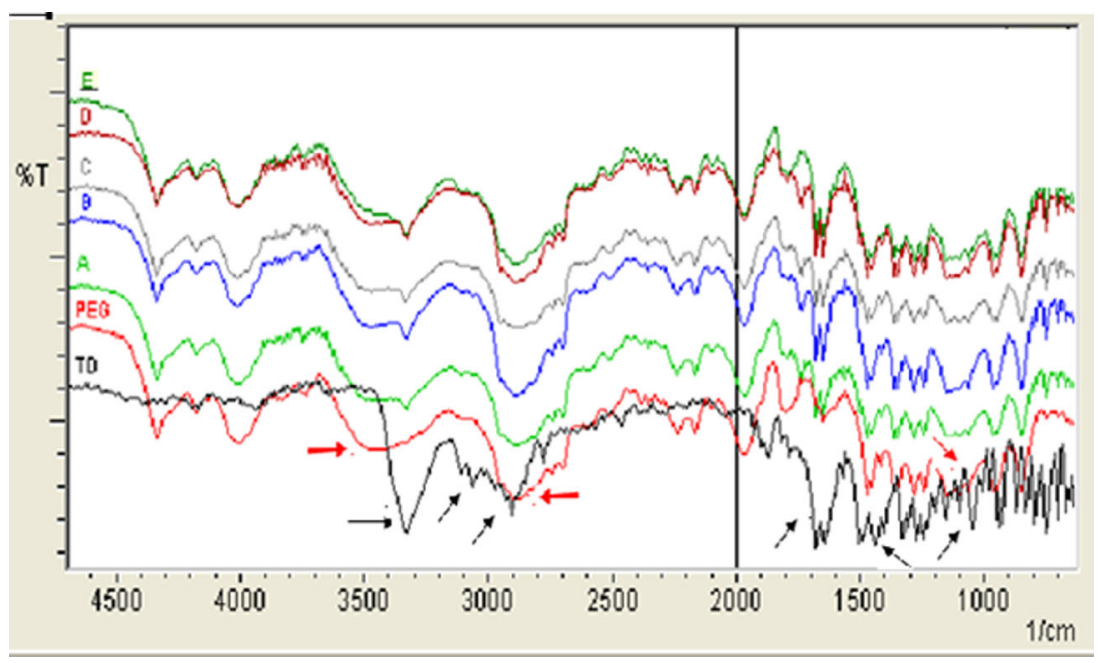

Fig. 6. FT-IR spectra of $A$ tablet formulation A, $B$ tablet formulation $\mathrm{B}, C$ tablet formulation $\mathrm{C}, D$ tablet formulation $\mathrm{D}$ and $E$ tablet formulation E; $T D$ Tadalafil powder, $P E G$ polyethylene glycol 4000

was dried either in oven at $40^{\circ} \mathrm{C}$ (formulation C) or via freeze dry (formulation D). After drying, all formulations were directly compressed as mentioned earlier in the experimental section. In addition, a fifth formulation, E, was prepared from physical mixture of Tadalafil powder and PEG and was directly compressed into tablets. The measured crushing strengths of tablet formulations showed slightly lower strengths for tablet formulations prepared using freeze-dried nanosuspensions. The measured crushing strengths $\left(K_{\mathrm{p}}\right)$ were $5.2 \pm 0.4,2.8 \pm 0.2,5.4 \pm 0.6$, $3.2 \pm 0.5$ and $3.7 \pm 0.3$ for formulations $\mathrm{A}, \mathrm{B}, \mathrm{C}, \mathrm{D}$ and $\mathrm{E}$, respectively. Dissolution of the tablets was conducted in a way similar to that used for Tadalafil nanosuspensions (in 1,000 $\mathrm{ml}$ of distilled water containing $0.3 \%$ SLS, at $100 \mathrm{rpm}$ and $37^{\circ} \mathrm{C}$ ). Results are shown in Fig. 5, where among all Tadalafil-PEG tablet formulations, formulation $\mathrm{B}$ showed the highest initial drug release rates in the initial phase (first 5-15 min) followed by formulation $\mathrm{A}$, while formulations $\mathrm{C}$ and $\mathrm{D}$ showed lower rates. This behaviour might be due to the fact that Tadalafil was initially prepared as nanosized particles in formulations $\mathrm{B}$ and A, while formulations $\mathrm{C}$ and $\mathrm{D}$ were obtained from Tadalafil raw powder suspension. Dissolution studies also showed that, in general, tablet formulations made of freeze-dried products; formulations $\mathrm{B}$ and $\mathrm{D}$ exhibited faster release rates during the initial times than the corresponding tablets made of oven-dried products: formulations $\mathrm{A}$ and $\mathrm{D}$, respectively. This may be explained by possible presence of Tadalafil crystals which exhibit higher rates of growth during drying in oven compared to freeze dry and therefore resulting to lower dissolution rates. This can also be the cause of the slightly higher crushing strengths of formulations $\mathrm{A}$ and $\mathrm{C}$ compared to formulations $\mathrm{B}$ and $\mathrm{D}$, which also contributed to the lower initial drug release rates of formulations $\mathrm{A}$ and $\mathrm{C}$. After the initial dissolution times,

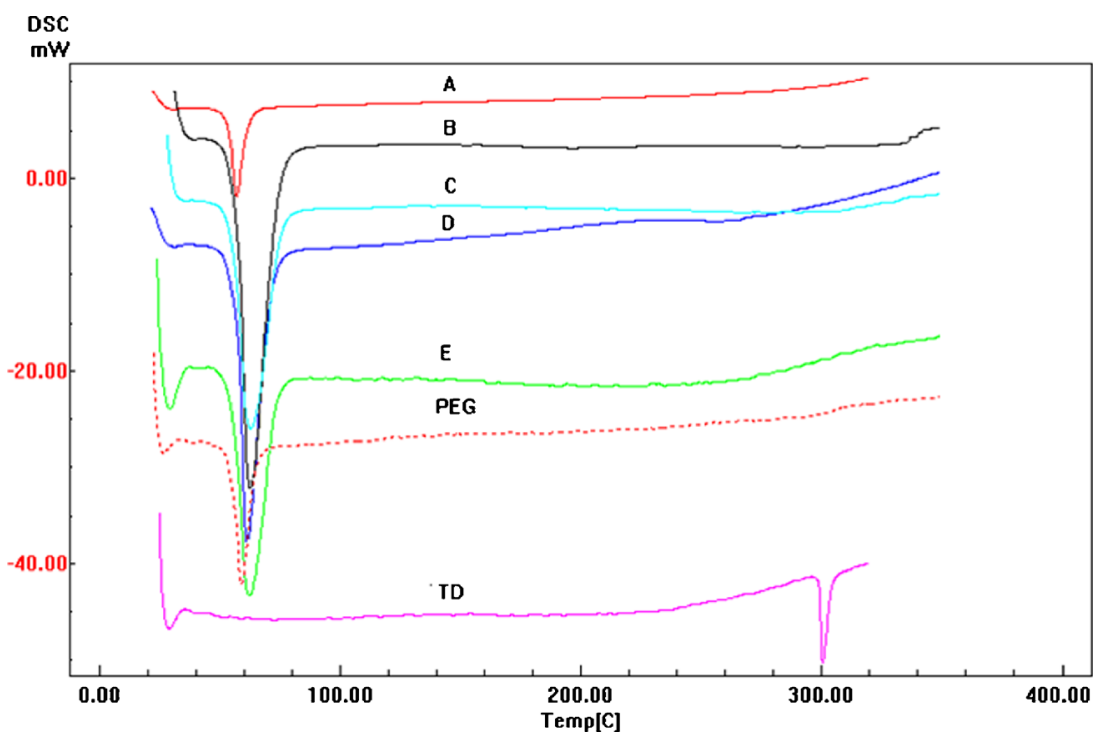

Fig. 7. DSC thermograms of $A$ tablet formulation $\mathrm{A}, B$ tablet formulation $\mathrm{B}, C$ tablet formulation $\mathrm{C}, D$ tablet formulation $\mathrm{D}$ and $E$ tablet formulation E; $T D$ tadalafil powder, $P E G$ polyethylene glycol 4000 
most of the drug was released at comparable release rates except for formulation $\mathrm{E}$ which, despites its relatively low crushing strength of $3.7 \pm 0.3$, showed the lowest release rates among all formulations since they were untreated physical mixture of the large particle size Tadalafil raw powder $(10 \mu \mathrm{m})$ and PEG 4000.

The FT-IR spectra of pure components and TadalafilPEG tablet formulations are shown in Fig. 6. The IR spectrum of Tadalafil is characterized by principal absorption peaks at $3,328 \mathrm{~cm}^{-1}$ due to $\mathrm{N}-\mathrm{H}$ stretching vibration and $3,092 \mathrm{~cm}^{-1}$ due to stretch of aromatic $\mathrm{C}-\mathrm{H}$. Two intense absorption bands attributed to amide $\mathrm{C}=\mathrm{O}$, and aromatic $\mathrm{C}=\mathrm{C}$ stretching vibrations were found at 1676 and $1649 \mathrm{~cm}^{-1}$ respectively. Other bands appeared at 2,905 $\mathrm{cm}^{-1}(\mathrm{C}-\mathrm{H}$ stretch, aliphatic $\mathrm{CH} 3$ ), 1,437.62 $\mathrm{cm}^{-1}$ (C-N stretch), 1,041 $\mathrm{cm}^{-1}$ (C-O-C stretch) and $745 \mathrm{~cm}^{-1}$ (benzene). PEG 4000 exhibited characteristic peaks at $2,890 \mathrm{~cm}^{-1}$ due to $\mathrm{C}-\mathrm{H}$ stretching, at $1,116 \mathrm{~cm}^{-1}$ due to $\mathrm{C}-\mathrm{O}$ stretching and at $3,350 \mathrm{~cm}^{-1}$ due to $\mathrm{O}-$ $\mathrm{H}$ stretching. Generally, the FTIR spectra of all tablet formulation products did not show significant changes with respect to the FTIR spectra of the pure components and in particular the characteristic bands of Tadalafil; the characteristic $-\mathrm{NH}$ stretching band at $3,328 \mathrm{~cm}^{-1}$, in addition to aromatic C-H stretch at $3,092 \mathrm{~cm}^{-1}$ and aliphatic $\mathrm{C}-\mathrm{H}$ stretch at $2,905 \mathrm{~cm}^{-1}$ of Tadalafil, was apparently masked in all the prepared systems by the broad intense band corresponding to the $\mathrm{OH}$ vibration at $3,350 \mathrm{~cm}^{-1}$ and by $\mathrm{C}-\mathrm{H}$ stretching at $2,890 \mathrm{~cm}^{-1}$. This could be due the low drug to PEG 4000 ratio $(6.67 \%, w / w)$. Therefore, we can assume absence of any chemical or physical interactions of the drug with the carrier. Similar results were reported for solid dispersions of Tadalafil using poloxamer 407 (36).

Figure 7 depicts the DSC thermograms of pure drug, PEG 4000 and Tadalafil-PEG tablet formulations. Tadalafil powder exhibited a single sharp endothermic peak at 301$302^{\circ} \mathrm{C}$. PEG 4000 showed an endothermic peak at $61^{\circ} \mathrm{C}$. The position of endothermic peaks of PEG 4000 was maintained in all tablet formulations A to E. However, Tadalafil endotherm disappeared in thermograms of all tablet formulations. This can be attributed to the formation of Tadalafil-PEG 4000 solid dispersion, and therefore, the existence of the drug in an amorphous form, or it can be due to the existence of drug crystals but in low concentrations $(6.67 \%, w / w)$ that were undetectable during DSC analysis as shown by formulation $\mathrm{E}$ (physical mixture) thermogram in Fig. 7. Another possibility is that PEG 400 melted first due to its low melting point, which then dissolved the drug on further heating.

When compared to Tadalafil nanosuspensions (TDTSp$\mathrm{OH}$ or TDTSp-N), Tadalafil-PEG formulations A and B showed lower rates of drug release. In addition to the enormous surface area of nanosuspensions relative to PEG tablets, this could be attributed to the lowered mobility of drug particles or molecules embedded within the PEG matrix system particularly in the initial phase. Furthermore, changes in the size of freeze-dried Tadalafil nanosuspension could have taken place and therefore affected the drug release rates. On the other hand, it was found that PEG 4000 matrices produced enhanced release rates of Tadalafil in formulations C, D and E compared to plain Tadalafil raw powder release rates conducted in SLS or in SLS/Tween80/Span80. This can be attributed to the formation Tadalafil-PEG solid dispersions in formulations $\mathrm{C}$ and $\mathrm{D}$, or to the hydrophilic effect of $\mathrm{PEG}$
4000 which exerted solubilization effect on Tadalafil powder during matrix dissolution of formulation $\mathrm{E}$.

Literature lacks any data about preparation of Tadalafil nanosuspensions. However, few studies exist adopting other methods to improve Tadalafil dissolution rates. Kumar et al. (37) showed that, at best, only $30-40 \%$ of Tadalafil were released during the first $5 \mathrm{~min}$ of dissolution and about $91 \%$ of cumulative drug release at the end of $90 \mathrm{~min}$ of dissolution of Tadalafil-PVP K30 and Tadalafil-PEG 6000 solid dispersions. Shaimaa et al. (38) reported a marked enhancement of Tadalafil dissolution rates via complexation using cyclodextrins where $75 \%$ of the drug were released in the first $5 \mathrm{~min}$. Therefore, our current method of development of Tadalafil nanosuspensions showed superiority in enhancement of dissolution rate of Tadalafil (more than $90 \%$ cumulative drug were released within the first $5 \mathrm{~min}$ ) compared to other employed methods.

\section{CONCLUSIONS}

Tadalafil nanosuspensions with adequate stability necessary for further processing were successfully prepared using combinations of nonionic surfactants comprised of Tween80 and Span80 at 1:1 ratio (in percent, weight/volume) with total concentration of $0.10 \%(w / v)$. Generally, the concentration of stabilizer employed to stabilize the nanosuspension has marked effect on the mean particle size; higher concentrations resulted in a smaller average particle size except in certain cases where particle size either increased with increasing stabilizer concentration such as Methocel E5 LV, Brij58, Cremophor EL and mixtures of Tween80/Cremophor EL or remained almost the same at all stabilizer concentrations such as $\mathrm{Na}$ docusate. The $\mathrm{pH}$ of the nanosuspension was a critical parameter affecting its stability, where adequate stability was obtained at $\mathrm{pH}$ 10. Tadalafil nanosuspensions prepared in alkaline media exhibited smaller mean particle size and therefore higher dissolution rates compared to those prepared in neutral media.

\section{ACKNOWLEDGMENTS}

Authors would like to acknowledge the support from TQ Pharma. Also, Dr. Abdallat Y., director of FFF project (JUJordan), is gratefully acknowledged.

\section{REFERENCES}

1. Lomabardino JG, Lowe III JA. A guide to drug discovery: the role of the medicinal chemist in drug discovery-then and now. Nat Rev Drug Discov. 2004;3:853-62.

2. Thomas R. Solubility enhancement with BASF pharma polymers. Lampertheim: BASF SE Pharma Ingredients \& Services; 2011.

3. Keck CM, Muller RH. Drug nanocrystals of poorly soluble drugs produced by high pressure homogenisation. Eur J Pharm Biopharm. 2006;62:3-16.

4. Lipinski C. Poor aqueous solubility - an industry wide problem in drug discovery. Am Pharm Rev. 2002;5:82-5.

5. Patro S, Himasankar K, Choudhury AA, Rao MEB. Effect of some hydrophilic polymers on dissolution rate of roxitromycin. Indian J Pharm Sci. 2005;67:334-41. 
6. Omaima AS, Mohammed AH, Nagia AM, Ahmed SZ. Formulation and optimization of mouth dissolve tablets containing rofecoxib solid dispersions. AAPS PharmSciTech. 2006;2:1-9.

7. Trivedi JS, Wells ML. In: Liu R, editor. Solubilization using cosolvent approach. Water-insoluble drug formulation. Denver: Interpharm; 2000. p. 141-68.

8. Gibaldi M. Biopharmaceutics and clinical pharmacokinetics, 4 ed. Hyderabad: Pharma Book Syndicate; 2008. p. 48.

9. Dressmann JB, Reppas C. In vitro-in vivo correlations for lipophilic, poorly water-soluble drugs. Eur J Pharm Sci. 2000;1:73-80.

10. Keck C, Fichtinger A, Viernstein H, Müller RH. Oral drug nanocrystals - effect of potential aggregation on bioavailability. Abstract, AAPS Annual Meeting, Baltimore; 2004.

11. Patravale VB, Date AA, Kulkarni RM. Nanosuspensions: a promising drug delivery strategy. J Pharm Pharmacol. 2004;56:827-40.

12. Wong M, Kellaway IW, Murdan S. Enhancement of the dissolution rate and oral absorption of a poorly water soluble drug by formation of surfactant-containing microparticles. Int J Pharm. 2006;317:61-8

13. Xia D, Quan P, Cui F. Preparation of stable nitrendipine nanosuspensions using the precipitation-ultrasonication method for enhancement of dissolution and oral bioavailability. Eur J Pharm Sci. 2010;40:325-34.

14. Liu D, Xu H, Tian B, Yuan K, Pan H, Ma S, et al. Fabrication of carvedilol nanosuspensions through the anti-solvent precipitation-ultrasonication method for the improvement of dissolution rate and oral bioavailability. AAPS PharmSciTech. 2012;13:295304.

15. Quan P, Xia D, Piao H, Piao H, Shi K, Jia Y, Cui F. Nitrendipine nanocrystals: its preparation, characterization, and in vitro-in vivo evaluation. AAPS PharmSciTech. 2011;12:1136-43.

16. List M, Sucker H. Hydrosols of pharmacologically active agents and their pharmaceutical compositions comprising them. US5389382; 1995

17. Guo Z, Zhang M, Li H, Wang E, Kougouslos E. Effect of ultrasound on antisolvent crystallization process. J Cryst Growth. 2005;273:555-63.

18. Louhi-Kultanen M, Karjalainen M, Rananen J, Huhtanen M, Kallas J. Crystallization of glycine with ultrasound. Int J Pharm. 2006;320:23-9.

19. Verma S, Gokhaleb R, Burgessa DJ. A comparative study of topdown and bottom-up approaches for the preparation of micro/ nanosuspensions. Int J Pharm. 2009;380:216-22.

20. Rabinow BE. Nanosuspensions in drug delivery. Nat Rev Drug Discov. 2004;3:785-96.

21. Porst H, Padma-Nathan H, Giuliano F, Anglin G, Varanese L, Rosen R. Efficacy of tadalafil for the treatment of erectile dysfunction at 24 and $36 \mathrm{~h}$ after dosing: a randomized controlled trial. Urology. 2003;62:121-6.

22. Anderson NR, Gullapalli RP. beta-Carboline pharmaceutical compositions. US6841167, 2001.

23. Hiemenz PC, Rajagopalan R. Principles of colloid and surface chemistry, 3rd ed. New York: Marcel-Dekker; 1997.

24. Caballero FG, Duran JGL. Suspension formulation. In: Nielloud F, editor. Pharmaceutical emulsions and suspensions. New York: Marcel Dekker; 2000. p. 127.

25. Kocbek P, Baumgartner S, Kristl J. Preparation and evaluation of nanosuspensions for enhancing the dissolution of poorly soluble drugs. Int J Pharm. 2006;312:179-86.

26. Merck index 2006, 14th ed. p. 1550-1.

27. Napper DH. Steric stabilization and the Hofmeister series. J Colloid Interface Sci. 1970;33:384-92.

28. Smitham JB, Napper DH. Steric stabilization in worse than $\theta$ solvents. Coll Polym Sci. 1979;257:748-56.

29. Rosen MJ. Surfactants and interfacial phenomena. New York: Wiley; 1989. p. 337.

30. ACD/PhysChem Suite. http://www.acdlabs.com/products/pcadmet/physchem/physchemsuite. 2012.

31. Lee RW, Shaw JM, McShane J, Wood RW. In: Liu R, editor. Particle size reduction. Water-insoluble drug formulation. Denver: Interpharm; 2000.

32. Liu R, Sadrzadeh N, Constantinides PP. In: Liu R, editor. Micellization and drug solubility enhancement. Water-insoluble drug formulation. Denver: Interpharm; 2000. p. 213-354.

33. Xu Q, Vasudevan TV, Somasundaran PJ. Adsorption of anionicnonionic and cationic-nonionic surfactant mixtures on kaolinite. Colloid Interface Sci. 1991;142:528-34.

34. Dongmei L, David GR. Mixed composition films of spans and Tween 80 at the air-water interface. Langmuir. 2000;16:8107-12.

35. Byron JP. Mixed surfactant systems to control dispersion stability in severe environments for enhancing chemical mechanical polishing (CMP) of metal surfaces. Ph.D. thesis, 2000.

36. Vyas V, Sancheti P, Karekar P. Physicochemical characterization of solid dispersion systems of tadalafil with poloxamer 407. Acta Pharm. 2009;59:453-61.

37. Ravi Kumar V, Sevukarajan M, Jayasri V, Pavankumar AG, Deepthi Y, Manjunath M, et al. Improvement of dissolution characteristics and bioavailability of tadalafil by solid dispersion technique using water-soluble polymers. Int J Adv Pharm. 2012;2(2):56-63.

38. Shaimaa MB-E, Seham AE, Mahmoud MG. Inclusion complexes of tadalafil with natural and chemically modified b-cyclodextrins. I: Preparation and in-vitro evaluation. Eur J Pharm Biopharm. 2008;70:819-27. 